Article

\title{
Acoustic Detection of Coronary Occlusions before and after Stent Placement Using an Electronic Stethoscope
}

\author{
Andrei Dragomir ${ }^{1}$, Allison Post ${ }^{1}$, Yasemin M. Akay ${ }^{1}$, Hani Jneid ${ }^{2,3}$, David Paniagua ${ }^{2,3}$, \\ Ali Denktas ${ }^{2,3}$, Biykem Bozkurt ${ }^{2,3}$ and Metin Akay ${ }^{1, *}$ \\ 1 Department of Biomedical Engineering, University of Houston, Houston, TX 77204, USA; \\ Andrei.Drag@gmail.com (A.D.); Allison.Post@central.uh.edu (A.P.); ymakay@uh.edu (Y.M.A.) \\ 2 Winters Center for Heart Failure Research, The Michael E. DeBakey VA Medical Center, Houston, TX 77030, \\ USA; jneid@bcm.edu (H.J.); dpaniagua@bcm.edu (D.P.); ali.denktas@bcm.edu (A.D.); \\ bbozkurt@bcm.edu (B.B.) \\ 3 Cardiovascular Research Institute, Baylor College of Medicine, Houston, TX 77030, USA \\ * Correspondence: makay@uh.edu; Tel.: +1-832-842-8860
}

Academic Editors: Raúl Alcaraz Martínez and Kevin H. Knuth

Received: 27 April 2016; Accepted: 23 July 2016; Published: 29 July 2016

\begin{abstract}
More than 370,000 Americans die every year from coronary artery disease (CAD). Early detection and treatment are crucial to reducing this number. Current diagnostic and disease-monitoring methods are invasive, costly, and time-consuming. Using an electronic stethoscope and spectral and nonlinear dynamics analysis of the recorded heart sound, we investigated the acoustic signature of CAD in subjects with only a single coronary occlusion before and after stent placement, as well as subjects with clinically normal coronary arteries. The CAD signature was evaluated by estimating power ratios of the total power above $150 \mathrm{~Hz}$ over the total power below $150 \mathrm{~Hz}$ of the FFT of the acoustic signal. Additionally, approximate entropy values were estimated to assess the differences induced by the stent placement procedure to the acoustic signature of the signals in the time domain. The groups were identified with this method with $82 \%$ sensitivity and $64 \%$ specificity (using the power ratio method) and $82 \%$ sensitivity and $55 \%$ specificity (using the approximate entropy). Power ratios and approximate entropy values after stent placement are not statistically different from those estimated from subjects with no coronary occlusions. Our approach demonstrates that the effect of stent placement on coronary occlusions can be monitored using an electronic stethoscope.
\end{abstract}

Keywords: coronary artery disease; stent; noninvasive monitoring; nonlinear dynamics analysis; approximate entropy

\section{Introduction}

Coronary artery disease (CAD) is a leading global cause of death, killing some 17.3 million people per year worldwide (a number that is projected to increase to 23.6 million by 2030), of which nearly 370,000 are Americans [1]. Including the cost of health care services, medications, and lost productivity, CAD alone cost the United States an estimated \$182 billion in 2015 [2]. CAD is characterized by atherosclerotic plaque formation resulting from complex cellular interactions in the intima of arteries with accumulation of fatty deposits, involvement of inflammatory mediators and endothelial dysfunction, and its precedence of restricted circulation to the heart muscle with risk of thrombosis. Total cessation of blood flow results in a myocardial infarction, which can lead to sudden death. Many patients are not aware they have CAD until their first heart attack [3]. Coronary angiography, the current standard of diagnosis and determination of severity of CAD, is expensive, risky and 
invasive [4]. Non-invasive and lower-risk modalities with varying degrees of accuracy and precision, such as coronary CT angiography, nuclear imaging, and cardiac MRI, although costly and associated with some risk, have been proposed for diagnosis and risk stratification of CAD [5,6]. There is a need for alternative affordable, low-risk, point-of-care modalities that are easily accessible to patients in rural communities where heart disease is prevalent and access to medical assessment is limited [7]. Bringing a cost-effective, portable, easy-to-use, low-risk diagnostic device to large, high-risk populations in need has the potential to save hundreds of thousands of lives each year.

The typical heart sounds "lubb, dub" are generated by blood colliding with the closed valves of the heart. The "lubb" sound, or the first heart sound (S1), is produced by the closing of the atrioventricular (AV) valves at the beginning of ventricular systole. This is the longest heart sound. The "dub" sound, or the second heart sound (S2), is produced by the closing of the semilunar valves at the end of ventricular systole. S2 is shorter than S1, and is followed by a pause. These sounds are typically clear and uninterrupted. Any disruption of these sounds, such a liquid rushing or gurgling, indicates a structural problem within the heart. Examples of recorded heart sounds are shown in Figure 1. Heart sounds S1 and S2 are audible using a simple stethoscope. The third and fourth heart sounds (S3 and $\mathrm{S} 4$, respectively) may be detected in some phonocardiogram recordings, provided the background noise is very small. S3 is caused by blood moving from the atria into the ventricles, and S4 occurs after the atria polarize, forcing more blood into the ventricles. Blood movement causes the walls of the heart to vibrate, causing low frequency sounds. These are hard to detect, as their magnitude is small compared to those of S1, S2 and background noise [8].
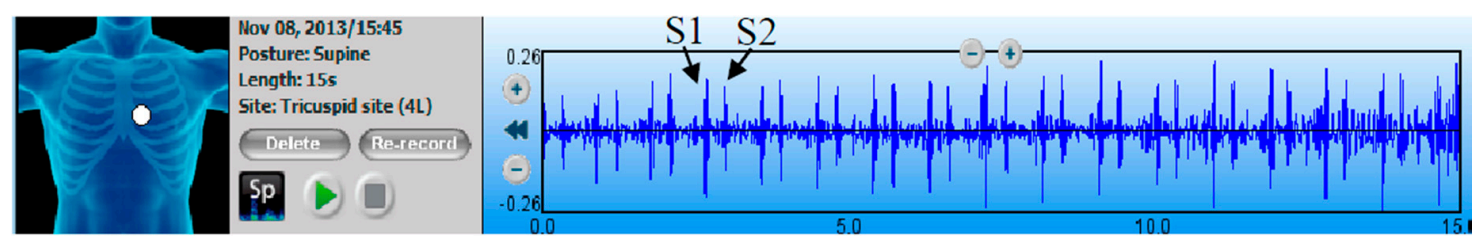

Figure 1. Patient recording as it appears in the 3M Littmann Steth-Assist Software (Version 1.2.963).

Coronary blood flow is affected by the arterial pressure, which can vary greatly. The pressure gradient and coronary blood flow usually depend on the contractility of the heart. Coronary blood flow characteristics through the coronary arteries are not the same, as flow through the left coronary artery is maximal during early diastole, whereas blood flow through the right coronary artery is maximal during peak systole [9].

The acoustic approach to diagnosing coronary artery disease relies on the principle that there are sounds associated with turbulent flow caused by partially occluded pipes. In this case, the partially occluded tubes are coronary arteries. However, the sounds associated with coronary artery disease are not easily detected with simple stethoscopes and the human ear. Instead, they require special instrumentation and/or signal processing in order to recognize the change between diseased and normal arteries [10-29]. This principle has been demonstrated in other vessels in the body, such as the bruits (sounds) associated with blocked carotid arteries, and the Korotkoff sounds that occur when the brachial artery is restricted by a pressure cuff and are used to measure blood pressure [15].

\subsection{Coronary Flow Analysis Techniques}

Many groups have pursued various analysis techniques in the quest for the acoustic signature of CAD. The first step investigators must take in searching for acoustic signatures of CAD in heart sound recordings is to find the ideal segment of the signal to analyze, which will contain sounds from the coronary arteries. Previous studies have shown the diastolic window to have the most promise. In the diastolic window, there is little extraneous noise and the signal-to-noise ratio is greater when searching for sounds associated with occluded coronary arteries $[16,20]$. 
In order to study the spectra of the heart sound recordings, the selected diastolic windows can be analyzed using fast Fourier transform (FFT). Studies by Akay et al. in canine femoral artery models confirmed the association of artery occlusion with increased energy in high frequencies [15]. In our previous study, we showed that normal and abnormal subjects can be discriminated using spectral analysis of the acoustic signals [29]. Spectral content estimated using the FFT was quantified and results suggested that diseased subjects consistently showed more energy content at high frequency. Other studies based on spectral features used various parametric models such as autoregression (AR), Multiple Signal Classification (MUSIC) algorithm [16,20], as well as empirical mode decomposition [30,31], wavelet analysis [24], or fast tracking filters [22,23].

In parallel, studies showed that the acoustic signature of CAD, being the product of the chaotic turbulence process, may exhibit signal complexity variations. Padmanabhan et al. [27] used fractal based analysis, Akay et al. [32] used approximate entropy, and Schmidt et al. [33] used both spectral and complexity-based acoustic features to discriminate between CAD and non-CAD patients. However, the above-mentioned studies did not investigate the effect of stent placement on the acoustic signatures of patients. Our ultimate goal is to define a framework for future longitudinal studies monitoring in-stent restenosis. In the present study, we aim to scrutinize whether the acoustic signature of CAD can be detected using spectral analysis and nonlinear analysis of heart sounds as a first step toward determining whether noninvasive assessment could be a suitable low-cost surrogate or complement for current imaging diagnostics preceding percutaneous coronary intervention.

\subsection{Study Design}

We focused on systematic CAD detection using commercially available electronic stethoscopes, which will allow for an expedited path to clinical use. The Littmann Electronic Stethoscope Model 3200 (3M, Saint Paul, MN, USA) with ambient noise reduction was used for this study, and shows promise as a useful tool in phonocardiology and CAD detection. The Littmann Model 3200 is widely available and well recognized by clinicians, and is the current standard of care in many clinical settings [34]. We have chosen to use this commercially available stethoscope to determine whether the unblocking effect of stent placement on coronary occlusions can be discriminated using signal processing and analysis of patient acoustic signature based on heart sound recordings before and after stent placement procedures. In this way, the patient can serve as his own control when comparing heart sounds of blocked and unblocked coronary arteries.

In summary, we implemented a study in clinical setting which would help identify the acoustic signature using an electronic stethoscope. We recorded acoustic signals from subjects with only a single coronary artery occlusion before and after stent placement at the Houston VA Hospital. We analyzed the recorded signals using spectral analysis techniques based on FFT by computing power ratios and comparing the results with those obtained using a nonlinear analysis technique based on approximate entropy. We then compared these recordings to the recordings of clinically normal patients with no coronary occlusions.

\section{Materials and Methods}

\subsection{Patient Inclusion and Exclusion Criteria}

Patients were screened and tested at the Michael E. DeBakey Houston VA Hospital in collaboration with the Winter Center for Cardiology Research of the Baylor Medical School. The DeBakey VA Hospital R\&D Committee and the Institutional Review Board (IRB) for Baylor College of Medicine and Affiliated Hospitals approved the study (protocol number H-32016). Study staff members who had completed the human subject protection educational requirements in compliance with all Federal regulations conducted informed consent negotiations. Prospective subjects were interviewed to determine preliminary eligibility. Informed consent was obtained, and subjects were enrolled in the study prior to any clinical testing, laboratory testing or intervention. Subjects were given a copy of 
the IRB approved consent form during the initial interview, and study staff explained to the subjects, in detail, the nature of the informed consent process, study purpose and procedures, time commitments, risks, potential benefits, treatment alternatives, rights as research participants, study staff contact information, confidentiality procedures, and arrangements for medical care provided in case of injury during the study. Subjects were given adequate time to consider their decision and encouraged to ask questions, both during the initial interview and throughout the study. Subjects were provided with a signed copy of the completed consent form.

The inclusion criteria for study patients were as follows: adult male patients with body mass index (BMI) less than 30, single lesion CAD, and patient consent for procedure. Patients were not enrolled in the study if they had one or more of the following exclusion criteria: heart valve disease, multi-vessel CAD, and implanted pacemaker or defibrillator. We enrolled patients with a low BMI in order to minimize the amount of fatty tissue between the heart and the electronic stethoscope, since the fatty tissue could muffle and attenuate the sounds in the recording. We excluded patients with valve disease, which can disrupt the duration and magnitude of S1 and S2. Leaky valves could also allow for regurgitation, which would cause high frequency noise in the diastolic window and interfere with our analysis of coronary artery sounds. Similarly, implanted pacemakers and defibrillators create noise, which would be difficult to eliminate from recordings. We focused on patients with single coronary artery occlusions. With only one physiological change occurring within the patient, the restoration of flow in one coronary artery, all other variables remain constant. Therefore, any change in sound theoretically only corresponds to the affected coronary artery. Based on these criteria, we tested 11 patients with CAD. The patients' disease state is listed in Table 1 below. Due to the skewed population at the VA Hospital, all patients included in the study are male. In order to compare the results with normal patients, we also obtained recordings from three patients who underwent coronary angiography at the VA Hospital and were found to have no coronary artery disease.

Table 1. Patient number and the corresponding coronary artery disease with percent stenosis. Abbreviations represent the following: Obtuse marginal (OM), Left Anterior descending (LAD), Circumflex (Cx), Postero-lateral artery (PLA), and Right coronary artery (RCA).

\begin{tabular}{cccc}
\hline \multicolumn{4}{c}{ Recruited Patient Information } \\
\hline Patient Number & Diseased Coronary & Percent Stenosis & Age \\
\hline 1 & OM2 & 90 & 82 \\
2 & Mid LAD & 50 & 57 \\
3 & Left Main & 70 & 62 \\
4 & Mid Cx & 90 & 68 \\
5 & Mid Cx & 80 & 70 \\
6 & Right PLA & 70 & 61 \\
7 & Mid LAD & 75 & 69 \\
8 & Mid LAD & 95 & 63 \\
9 & Proximal LAD & 95 & 84 \\
10 & Mid RCA & 80 & 67 \\
11 & Mid Cx & 80 & 72 \\
\hline
\end{tabular}

\subsection{Data Recording}

Heart sounds were recorded on the day of the procedure, before and after stent placement. The stethoscope was set to diaphragm mode, which amplifies sounds from $20 \mathrm{~Hz}$ to $2000 \mathrm{~Hz}$, and emphasizes sounds between $100 \mathrm{~Hz}$ and $500 \mathrm{~Hz}$-our spectra of interest. The stethoscope was placed at the 4 th intercostal space, $6-8 \mathrm{~cm}$ to the right of the midline of the sternum, and held in place for the duration of the recording. Sound was recorded for $15 \mathrm{~s}$ while the patient held his breath to reduce chest cavity noise. Patients were in the supine position. 


\subsection{Diastolic Window Determination}

For each cardiac cycle, a diastolic window was selected 100 milliseconds after the end of S2 to avoid signal contamination by valve or regurgitation sounds (shown in Figure 2). Windows of 128 milliseconds in length were selected for analysis. This time segment corresponds with heavy coronary artery flow while avoiding other heart sound noise, as detailed in previous studies [29,32]. The signal was sampled at a rate of $4 \mathrm{kHz}$.

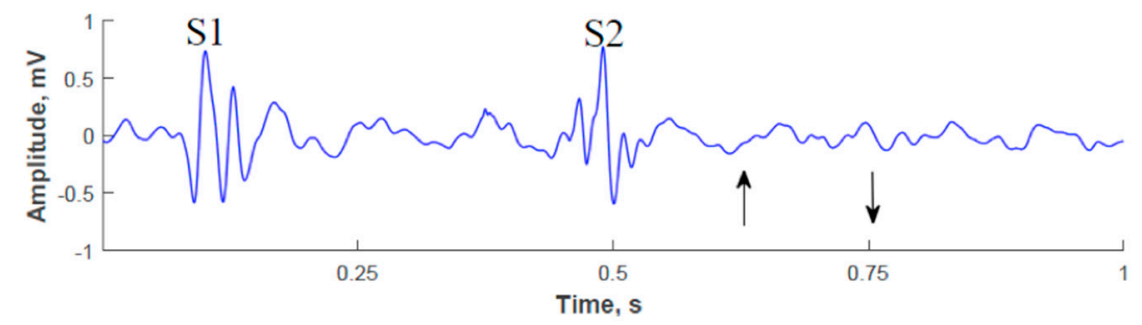

Figure 2. Determining the diastolic window in a cardiac cycle. Figure shows the raw signal from a normal patient. Arrows indicate the beginning and the end of the selected segment.

\subsection{Filtering}

First, the signal was detrended using the MATLAB (Version R2014a) detrend function. When detrending the signal, a linear trend that best fits the data in the least squares sense was subtracted, creating a flat baseline for the data. In order to normalize the signal, the root mean square (RMS) was calculated in the time domain for each recording, and dividing by the RMS normalized the signals. Once the signal was detrended and RMS-normalized, a bandpass filter was applied. A 5th order Butterworth bandpass filter was applied with a low frequency cutoff at $60 \mathrm{~Hz}$ and a high frequency cutoff at $500 \mathrm{~Hz}$. In Butterworth filters, the frequency response is maximally flat in the bandpass region and rolls off toward zero in the stopband. The 5 th order gives a sharp cut off for bandpass filtering without disrupting the phase of the signal.

\subsection{Spectral Analysis}

The FFT of each diastolic window was estimated for spectral analysis. We identified 10 segments for each patient pre-stent placement and 10 segments for each patient post-stent placement. Representative FFT spectra of a diastolic window from the pre-stent recording and a diastolic window from the post-stent recording are shown in Figure 3.

In order to compare the FFT of each diastolic window before and after stent placement, a ratio of the power above and below a frequency threshold was determined. The area below the FFT curve above threshold represents the total power above $150 \mathrm{~Hz}$, A. Frequencies above the threshold have been shown in previous studies to have changes between normal and diseased patients $[21,33]$. The area below the FFT curve below threshold, B, represents the total energy below $150 \mathrm{~Hz}$. The ratio A/B was used to differentiate between occluded and non-occluded flow. A larger power ratio indicated disease, while smaller power ratio indicates normal physiology. The power ratio is represented as:

$$
r=\frac{A}{B}
$$


(a) Before Stent

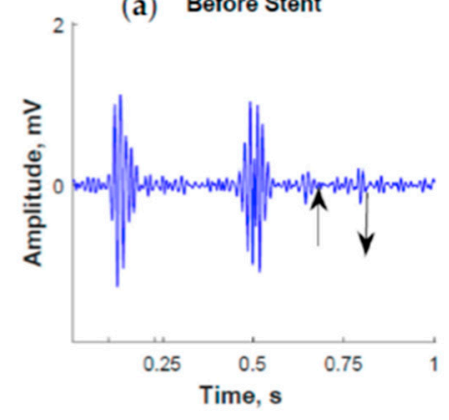

(d)

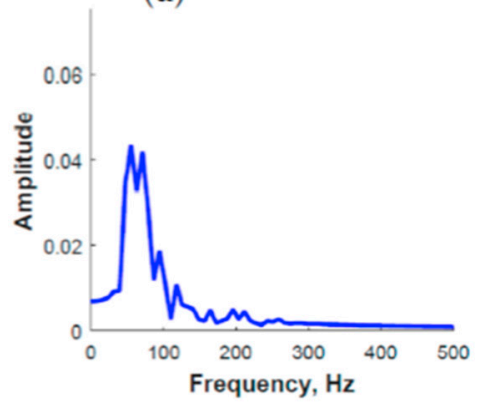

(b) After Stent

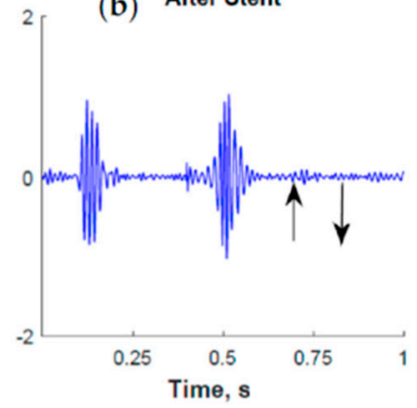

(e)

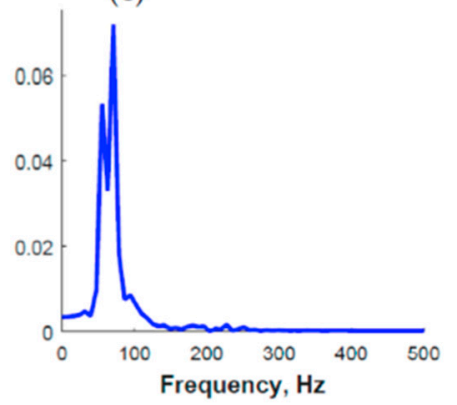

(c) Normal Patient

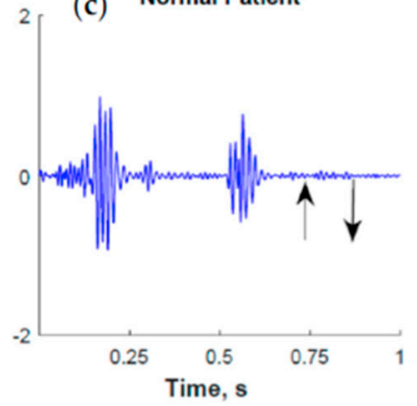

(f)

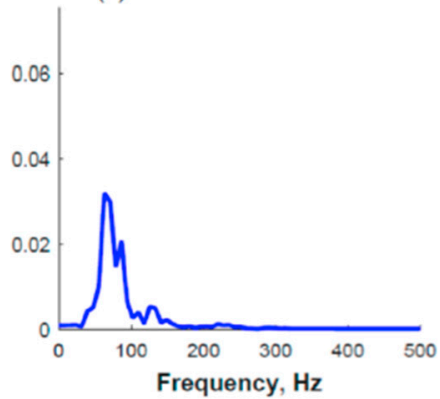

Figure 3. Cardiac cycles signal before (a) and after (b) stent placement, as well as for a clinically normal patient (c) with the diastolic window identified. FFT spectra corresponding to the windows shown above $(\mathbf{d}-\mathbf{f})$. Before and after stent placement data is from Patient 3.

\subsection{Approximate Entropy}

The time-domain preprocessed diastolic segments were then analyzed using the approximate entropy method (ApEn), a nonlinear dynamics measure commonly used to quantify the complexity of a time-series. ApEn produces a model-independent measure of signal entropy. It summarizes a time series signal into a non-negative numerical value, with more random signals having higher ApEn values, which generally reflect increased complexity in their generating systems. In other words, ApEn estimates the logarithmic likelihood that successive runs of temporal patterns that are close within a certain threshold, $r$, over a defined number of observations, $m$, remain close in the next incremental comparisons. A lower likelihood for these segments of remaining close within the defined threshold produces high ApEn values, indicating a higher level of entropy in the signal.

Briefly, ApEn estimates work with segments of length $m$, defined as $X(i)=[x(i), \ldots, x(i+m-1)]$. Distances between segments $X(i)$ and $X(j)$ can be estimated as:

$$
d[X(i), X(j)]=\max _{k=0, m-1}[|x(i+k)|-x(j+k)] \leqslant r
$$

For any given $X(i)$, the ratio of the number of vectors $X(j)$ for which the distances between $X(i)$ and $X(j)$ smaller than the threshold, $r,\left(N_{r}^{m}\right)$ to the total number of segments $N-m+1$ can be calculated as:

$$
C_{r}^{m}(i)=\frac{N_{r}^{m}(i)}{N-m+1} \text { for } i=1, \ldots, N-m+1
$$

Subsequently, the approximate entropy is estimated as a function of segment length $m$, and threshold, $r$ :

$$
\operatorname{ApEn}(m, r)=\lim _{N \rightarrow \infty}\left[\Phi^{m}(r)-\Phi^{m+1}(r)\right]
$$

where:

$$
\Phi^{m}(r)=\sum_{i=1}^{N-m+1} \ln \left(\frac{C_{r}^{m}(i)}{N-m-1}\right)
$$


ApEn was shown to be particularly efficient in the case of short data segments, and is less sensitive to noise and outliers than other regularity statistics [35]. Moreover, it can be used in the analysis of both stochastic and deterministic signals [36,37]. In the present study, the parameter values we used are $m=2$ and $r=0.1 \mathrm{SD}(x(i))$, where $\mathrm{SD}(x(i))$ represents the standard deviation of the isolated diastolic segment. Based on our previous studies and the seminal paper by Pincus [35,36], $m=2$ is the most commonly used value for ApEn analysis. As indicated in Section 2.6, we chose the same value for $\mathrm{m}$ based on our previous work, and on published work using ApEn on biosignals [37-39]. As highlighted by Pincus, the utility of ApEn is represented by it being a relative, not absolute, measure. Therefore, various values of $m$ and $r$ do not yield significant differences. Additional details on the ApEn method and its implementation are given elsewhere [37-39].

\section{Results}

Based on the inclusion and exclusion criteria detailed in the Materials and Methods section, 11 patients were enrolled in the study. Figure 3 illustrates representative examples of selected diastolic segments from a patient before and after the stent placement procedure (Figure $3 \mathrm{a}, \mathrm{b}$, respectively) and from a clinically normal patient (Figure 3c). The lower panels (Figure 3d-f) present the corresponding FFT spectra estimated for these segments. We observed that not only was there a decrease in the power of the high-energy frequencies $(150 \mathrm{~Hz}$ or greater) from before stent to after stent, there was also an increase in the low frequency energy (below $150 \mathrm{~Hz}$ ) in some patients' FFT spectral analysis. An example of both phenomena is visible in Figure 3d,e. This increase in low frequency is most likely attributed to the improvement in heart function after stent placement. When blood flow is restored to the coronary artery, oxygen is again brought to the ischemic area of the heart. The heart muscle can then contribute to the contraction of the heart during the cardiac cycle, increasing the ejection fraction of the blood and therefore increasing the pressure on the valves when closing. The closing sounds may then become stronger, adding low frequency content to the heart sound recording. All power ratio values were averaged across patients to give an average pre-stent ratio and an average post-stent ratio value. Ratios for all patients were then compared using two-way ANOVA.

In most patients, the power ratios and ApEn values both showed a downward trend between preand post-stent recordings. A special case was that of Patient 2, who showed a significant increase in the ApEn values (and power ratios) from pre- to post-stent, going against the established trend for the data. This is most likely due to the presence of a heart murmur associated with a leaky valve, which was not diagnosed until after the data was collected. Additionally, the lesion repaired was a borderline $50 \%$ stenosis, which could affect the analysis. Therefore, Patient 2's trend opposition is not unexpected.

Figure 4 shows the overall results for CAD patients (before and after stent placement procedure), as well as for clinically normal patients. Average power ratio values obtained from the spectral analysis are presented on the left panel (Figure 4a) and average ApEn values obtained from the nonlinear dynamics analysis are displayed on the right panel (Figure $4 \mathrm{~b}$ ). Healthy patients (patients who underwent coronary angiography and were found to have normal coronaries at the VA hospital) serve as reference in our study. The ApEn values and power ratios of healthy subjects' recordings are expected to be lower than those of pre-stent of study patients' recordings.

The pre-stent ApEn values were significantly higher ( $p<0.05$, two-way ANOVA) than those estimated from data recorded post-stent. These results indicate the presence of a high nonlinear component in the time-series diastolic heart sounds data segments in the diseased patients, highlighting the higher complexity of the underlying system generating these sounds. As expected, we did not observe significant differences between data recorded from clinically normal patients and data recorded from diseased patients post-stent. Patients with clinically normal coronary physiology have smooth blood flow through these arteries, and therefore should not exhibit complex patterns of variation during the diastolic period. When comparing ApEn values of clinically normal patients with those of pre-stent group, the values were significantly different $(p<0.05$, unpaired Welch test). 


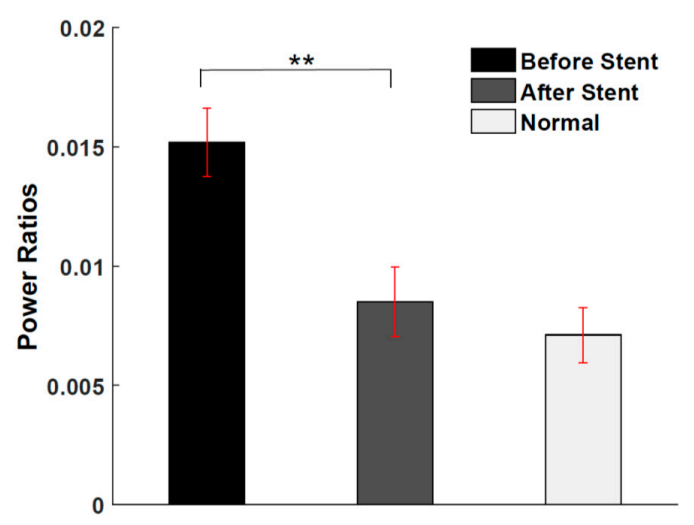

(a)

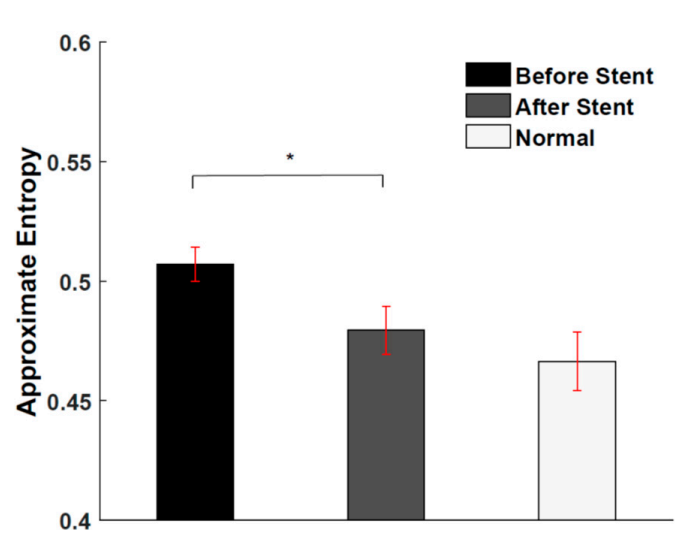

(b)

Figure 4. (a) Power ratios and (b) approximate entropy values of diastolic segments from all CAD patients, before and after stent placement, and clinically normal patients (mean \pm standard error). * denotes statistical significance $(p<0.05) .{ }^{* *}$ denotes statistical significance $(p<0.01)$.

By comparison, results of the spectral analysis confirm the results of the nonlinear analysis. The power ratios for the diseased subjects are significantly lower after stent placement $(p<0.01)$. Additionally, the recordings of post-stent patients are not statistically different from the clinically normal patients. Similarly with nonlinear dynamics analysis results, power ratios of clinically normal patients were significantly lower when compared to those of the pre-stent group $(p<0.01$, unpaired Welch test).

\section{Receiver Operating Characteristic}

Receiver Operating Characteristic (ROC) curves are shown in Figure 5 for power ratios (a) and ApEn (b). Using all 11 patients and their before and after stent power ratios, we devised a leave-one-out validation framework in conjunction with linear discriminant (LDA) classifiers. Specifically, we repeatedly trained LDA classifiers on all but one before and after stent power ratios and tested the classifier on the withheld power ratio sample. The same procedure was repeated for ApEn values. Using this framework, we obtained an overall efficiency of $73 \%$ for power ratios, with $82 \%$ specificity, $64 \%$ sensitivity and an area under curve (AUC) value of 0.8347 . For ApEn, the overall efficiency was lower, $68 \%$, with $82 \%$ specificity, $55 \%$ sensitivity and AUC $=0.7430$. The optimal cut-offs for maximizing the sensitivity and specificity were 0.014 for power ratios and 0.5 for ApEn.

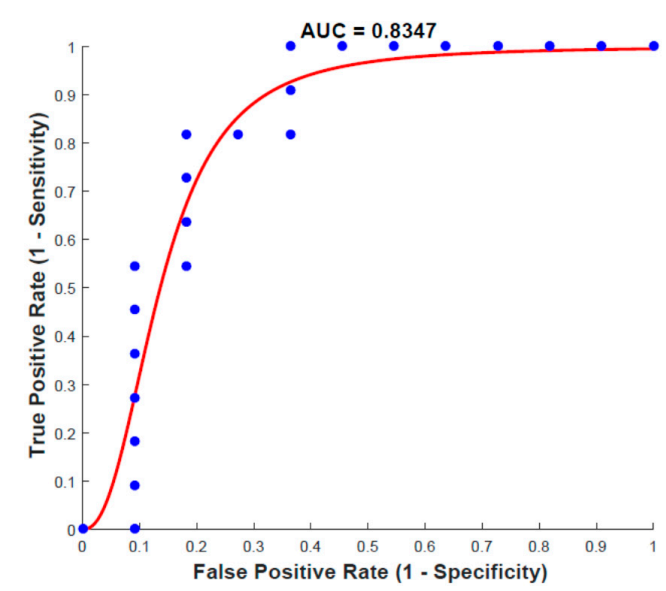

(a)

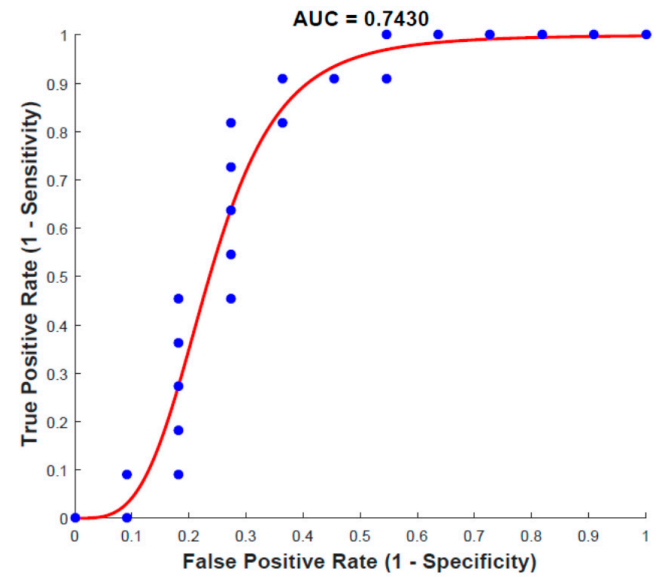

(b)

Figure 5. ROC curves obtained for power ratios (a) and ApEn values (b). 


\section{Discussion and Conclusions}

Previous studies suggested that occluded coronary arteries produce turbulent flow associated with coronary artery disease. If these sounds can be identified, they can be used as a clinical diagnostic tool. In this study, we proposed to use the electronic stethoscope to evaluate this hypothesis. Preceding studies investigated differences in heart sounds produced by coronary turbulent flow in normal and CAD patients. Our present work investigates whether the differences between the acoustic signatures of CAD patients, before and after stent placement, are detectable, and therefore whether such a noninvasive approach can be used to monitor the unblocking effect of stent placement. We believe our approach complements the expensive imaging-based diagnostic approaches, which are only available in specialized clinical departments, and could be used as an effective early diagnosis or screening tool before invasive diagnosis, as well as an efficient monitoring tool for longitudinal observation of restenosis development after stent placement.

The results above confirm previous study findings that power ratios determined from FFT of acoustic signals recorded with an electronic stethoscope are an effective combination for distinguishing between occluded and non-occluded coronary artery flow. The pre-stent power ratios in this study are statistically larger than the post-stent ratios. These results were confirmed with a nonlinear analysis method based on estimating approximate entropy values of the recorded signals, however, in the present study, the power ratio-based method showed a slightly higher discriminative power than the ApEn based method.

In order to effectively assess stent placement, there must be a precise method for distinguishing coronary arteries with occlusions from coronary arteries without occlusions. Using the 3M Littmann Model 3200 electronic stethoscope to record heart sounds and FFT to analyze the power of the spectra in those recordings, we were able to successfully distinguish diseased from non-diseased subjects using the calculated power ratios for each group with an overall efficiency of $73 \%$, even including the second subject who was later diagnosed with a heart murmur. Though the sample group is small $(n=11)$, the results hold promise for larger future studies. A similar trend in the results was observed using the ApEn method, which indicated increased complexity in the diastolic segments recorded from disease patients before stent placement. A sensitivity of $82 \%$ and a specificity of $55 \%$ were obtained using the ApEn method.

In our study, pre-stent power ratios and approximate entropy values were significantly higher in patients with obstructive coronary lesions than in clinically normal patients; the same patients' post-stent values appeared to be restored to levels similar to those of patients without obstructive coronary artery disease. The similarity in the spectral content and approximate entropy values implies similar sound profiles and similar acoustic signatures. Because the clinically normal patients have unobstructed flow in the coronary arteries, the similar lower values in the post-stent group implies that the flow is again unimpeded and has returned to a normal flow state.

Because acoustical parameters after stent placement are not statistically different from those estimated from healthy subjects with no coronary occlusions, our approach demonstrates that the effectiveness of stent placement can be traced or monitored using an electronic stethoscope. By establishing a baseline average in the approximate entropy value (or power ratio) in normal senior subjects, we can compare other subjects' values to the normal values to determine whether or not an occlusion or flow disturbance is still present. An average approximate entropy value (or power ratio) that is statistically higher than normal would indicate there is still an obstruction in the flow, indicating a need for further intervention or treatment. The normal group for this study was small and only included three subjects. A much larger group of normal subjects is needed for comparison to healthy controls. This is a proof-of-principle preliminary study intended to show the efficacy of the platform in clinics. It is extremely challenging to find patients with only one occlusion, since almost all patients will have multiple occlusions with different levels of severity. However, it is crucial to show the changes in acoustical parameters before and after stent placement, since we can relate these changes to the modification of the stenosis. 
Although our preliminary data supports that our platform can detect whether or not a patient is in need of a stent placement, our ultimate goal is to use this approach as a noninvasive method to assess for restenosis and/or development of de novo stenotic coronary lesions using acoustical parameters, including entropy and the determined power ratios. We plan to record data from unsuccessful stent placements in the near future for comparison. We also plan to include more subjects, including those with less than $50 \%$ occlusion, to strengthen the statistical power and further investigate the computational accuracy of our study. Additionally, we aim to improve recording quality by reducing noise in the recording room This would require patient isolation in a room with little or no electromagnetic interference, as various sources in hospital emergency units, such as two-way radios and medical telemetry equipment, may provide electromagnetic interference in the frequency band in which we are focusing [40]. We also aim to improve result accuracy by implementing a framework for reducing variability in stethoscope displacements. Furthermore, implementation of a multichannel recording system would provide additional acoustic signature data and help to localize the lesion.

Once the CAD acoustic signature threshold has been optimized, the system could easily be implemented in a clinical setting, providing a simple, inexpensive, and accurate screening tool for CAD or for stent restenosis in the future.

Acknowledgments: This study was supported by the United States National Science Foundation (CBET-0828912). No funds are available for covering the costs to publish in open access. The authors also appreciate the efforts of Kathryn Mossman for professional editing.

Author Contributions: Metin Akay, Biykem Bozkurt and Yasemin M. Akay conceived and designed the experiments and helped to write the paper; Allison Post recorded the data for the experiments and helped with data analysis and writing the paper; Andrei Dragomir analyzed the data and helped with writing the paper; Hani Jneid, David Paniagua, and Ali Denktas helped with data recording and provided clinical interpretation of the data. All authors have read and approved the final manuscript.

Conflicts of Interest: The authors declare no conflict of interest.

\section{References}

1. Mozaffarian, D.; Benjamin, E.J.; Go, A.S.; Arnett, D.K.; Blaha, M.J.; Cushman, M.; Das, S.R.; de Ferranti, S.; Després, J.P.; Fullert, H.J.; et al. Executive Summary: Heart Disease and Stroke Statistics—2016 Update: A Report from the American Heart Association. Circulation 2016, 133, 447-454. [CrossRef] [PubMed]

2. American Heart Association. Heart Disease and Stroke Statistics 2016 Update. Available online: http://www.heart.org/idc/groups/ahamah-public/@wcm/@sop/@smd/documents/downloadable/ ucm_480110.pdf (accessed on 26 July 2016).

3. Heart Disease Facts. Centers for Disease Control and Prevention. Available online: http://www.cdc.gov/ heartdisease/facts.htm (accessed on 26 July 2016).

4. Libby, P. Inflammation in Atherosclerosis. Nature 2002, 420, 868-874. [CrossRef] [PubMed]

5. Achenbach, S.; Marwan, M.; Ropers, D.; Schepis, T.; Pflederer, T.; Anders, K.; Kuettner, A.; Daniel, W.G.; Uder, M.; Lell, M.M. Coronary computed tomography angiography with a consistent dose below $1 \mathrm{mSv}$ using prospectively electrocardiogram-triggered high-pitch spiral acquisition. Eur. Heart J. 2010, 31, 340-346. [CrossRef] [PubMed]

6. De Graaf, F.R.; Schuijf, J.D.; van Velzen, J.E.; Kroft, L.J.; de Roos, A.; Reiber, J.H.; Boersma, E.; Schalij, M.J.; Spanó, F.; Jukema, J.W.; et al. Diagnostic accuracy of 320-row multidetector computed tomography coronary angiography in the non-invasive evaluation of significant coronary artery disease. Eur. Heart J. 2010, 31, 1908-1915. [CrossRef] [PubMed]

7. Turchetti, G.; Lorenzoni, V.; Bellelli, S.; Pierotti, F.; Rovai, D.; Caselli, C.; Underwood, S.R.; Knuuti, J.; Neglia, D. Cost-Effectiveness of Different Strategies for the Diagnosis of Stable Coronary Artery Disease: Results from the Evinci Study. Circulation 2014, 130 (Suppl. 2), A15359. [CrossRef] [PubMed]

8. Lanzer, P. Mastering Endovascular Techniques: A Guide to Excellence; Lippincott Williams \& Wilkins: Philadelphia, PA, USA, 2007.

9. Ramanathan, T.; Skinner, H. Coronary Blood Flow. Oxf. J. 2005, 5, 61-64.

10. Padmanabhan, V.; Semmlow, J.; Welkowitz, W. Accelerometer Type Cardiac Transducer for Detection of Low Level Heart Sound. IEEE Trans. Biomed. Eng. 1993, 40, 21-28. [CrossRef] [PubMed] 
11. Murrill, S.R.; Scanlon, M.V. Design for Heart Sound Extraction Algorithm for an Acoustic Based Health Monitoring System. Available online: http://www.arl.army.mil/arlreports/2002/ARL-MR-517.pdf (accessed on 26 July 2016).

12. Kroli, M.W. Heart Sound Sensor. U.S. Patent 4,672,976, 16 June 1987.

13. Schwartz, R.S.; Reeves, J.T.; Sodal, I.E.; Barnes, F.S. Improved Phonocardiogram System Based on Acoustic Impedance Matching. Am. J. Physiol. 1980, 238, H604-H609. [PubMed]

14. Guler, I.; Kiymik, M.K.; Fuler, N.F. Order Determination in Autoregressive Modeling of Diastolic Heart Sounds. J. Med. Syst. 1996, 20, 11-17. [CrossRef] [PubMed]

15. Akay, Y.M.; Akay, M.; Welkowitz, W.; Lewkowicz, S.; Palti, Y. Dynamics of Sounds Caused by Partially Occluded Femoral Arteries in Dogs. Ann. Biomed. Eng. 1994, 22, 493-500. [CrossRef] [PubMed]

16. Semmlow, J.; Akay, M.; Welkowitz, W. Noninvasive Detection of Coronary Artery Disease Using Parametric Spectral Analysis Methods. IEEE Eng. Med. Biol. Mag. 1990, 9, 33-36. [CrossRef] [PubMed]

17. Akay, M.; Welkowitz, W.; Semmlow, J.L.; Kostis, J. Application of the ARMA Method to Acoustic Detection of Coronary Artery Disease. Med. Biol. Eng. Comput. 1991, 29, 365-372. [CrossRef] [PubMed]

18. Akay, M.; Akay, Y.M.; Welkowitz, W.; Semmlow, J.; Kostis, J. Application of Adaptive Filters to Noninvasive Acoustical Detection of Coronary Occlusions before and after Angioplasty. IEEE Trans. Biomed. Eng. 1992, 39, 176-184. [CrossRef] [PubMed]

19. Semmlow, J. Biosignal and Biomedical Image Processing: MATLAB-Based Applications; Marcel Dekker: New York, NY, USA, 2004.

20. Akay, M.; Semmlow, J.; Welkowitz, W.; Bauer, M.; Kostis, J. Noninvasive Detection of Coronary Stenosis before and after Angioplasty Using Eigen Vector Methods. IEEE Trans. Biomed. Eng. 1990, 37, 1095-1104. [CrossRef] [PubMed]

21. Akay, Y.M.; Akay, M.; Welkowits, W.; Semmlow, J.; Kostis, J. Noninvasive Acoustical Detection of Coronary Artery Disease: A Comparative Study of Signal Processing Methods. IEEE Trans. Biomed. Eng. 1993, 40, 571-578. [CrossRef] [PubMed]

22. Akay, M.; Akay, Y.M.; Welkowitz, W.; Semmlow, J.L.; Kostis, J. Application of Adaptive FFT/FAST Zero Tracking Filters to Noninvasive Characterization of the Sound Pattern Caused By Coronary Artery Stenosis Before and After Angioplasty. Ann. Biomed. Eng. 1993, 21, 9-17. [CrossRef] [PubMed]

23. Akay, M.; Akay, Y.M.; Welkowitz, W.; Semmlow, J.L.; Kostis, J. Noninvasive Characterization of the Sound Pattern Caused by Coronary Artery Stenosis Using FTF/FAEST Zero Tracking Filters: Normal/Abnormal Study. Ann. Biomed. Eng. 1993, 21, 175-182. [CrossRef] [PubMed]

24. Akay, Y.M.; Akay, M.; Welkowits, W.; Kostis, J. Noninvasive Detection of Coronary Artery Disease Using Wavelet-Based Fuzzy Neural Networks. IEEE Eng. Med. Biol. Mag. 1994, 13, 761-764. [CrossRef]

25. Akay, M. Noninvasive Diagnosis of Coronary Artery Disease Using a Neural Network Algorithm. Biol. Cybern. 1992, 67, 361-367. [CrossRef] [PubMed]

26. Akay, M.; Welkowitz, W. Acoustical Detection of Coronary Occlusions Using Neural Networks. J. Biomed. Eng. 1993, 15, 469-673. [CrossRef]

27. Padmanabhan, V.; Semmlow, J.L. Dynamical analysis of diastolic heart sounds associated with coronary artery disease. Ann. Biomed. Eng. 1994, 22, 264-271. [CrossRef] [PubMed]

28. Semmlow, J.; Rahalkar, K. Acoustic Detection of Coronary Artery Disease. Annu. Rev. Biomed. Eng. 2007, 9, 449-469. [CrossRef] [PubMed]

29. Gauthier, D. Detection of Coronary Artery Disease Using an Electronic Stethoscope. MSc Thesis, Arizona State University, Tempe, AZ, USA, 2007.

30. Zhao, Z.D. Instantaneous Frequency Analysis of Diastolic Murmurs for Coronary Artery Disease. In Proceedings of the International Conference on Neural Networks and Brain, Beijing, China, 13-15 October 2015; Volume 2, pp. 1097-1100.

31. Schmidt, S.E.; Hols-Hansen, C.; Graff, C.; Toft, E.; Struijk, J.J. Detection of Coronary Artery Disease with an Electronic Stethoscope. In Proceedings of the Computers in Cardiology, Durham, NC, USA, 30 September-3 Octorber 2007; pp. 757-760.

32. Akay, M.; Akay, Y.M.; Gauthier, D.; Paden, R.G.; Pavlicek, W.; Fortuin, F.D.; Sweeney, J.P.; Lee, R.W. Dynamics of diastolic sounds caused by partially occluded coronary arteries. Biomed. Eng. IEEE Trans. 2009, 56, 513-517. [CrossRef] [PubMed] 
33. Schmidt, S.E.; Hols-Hansen, C.; Graff, C.; Toft, E.; Struijk, J.J. Acoustic features for the identification of coronary artery disease. Biomed. Eng. IEEE Trans. 2015, 62, 2611-2619. [CrossRef] [PubMed]

34. 3M Littman Electronic Stethoscope Model 3200. Available online: http://www.littmann.com/wps/portal/ 3M/en_US/3M-Littmann/stethoscope/stethoscope-catalog/catalog/?N=5002684+5932256\&rt=c3 (accessed on 26 July 2016).

35. Pincus, S.M. Approximate entropy as a measure of system complexity. Proc. Natl. Acad. Sci. USA 1991, 88, 2297-2301. [CrossRef] [PubMed]

36. Pincus, M.S.; Gladstone, I.M.; Ehrenkranz, R.A. A regularity statistic for medical data analysis. J. Clin. Monit. 1991, 7, 335-345. [CrossRef] [PubMed]

37. Srinivasan, V.; Eswaran, C.; Sriraam, N. Approximate Entropy-Based Epileptic EEG Detection Using Artificial Neural Networks. IEEE Trans. Inform. Technol. Biomed. 2007, 11, 288-295. [CrossRef]

38. Lu, S.; Chen, X.; Kanters, J.K.; Solomon, I.C.; Chon, K.H. Automatic selection of the threshold value $r$ for approximate entropy. IEEE Trans. Biomed. Eng. 2008, 55, 1966-1972. [PubMed]

39. Dragomir, A.; Akay, Y.M.; Curran, A.K.; Akay, M. Complexity measures of the central respiratory networks during wakefulness and sleep. J. Neural Eng. 2008, 5, 254-261. [CrossRef] [PubMed]

40. Lapinsky, S.E.; Easty, A.C. Electromagnetic Interference in Critical Care. J. Crit. Care 2006, 21, $267-270$. [CrossRef] [PubMed]

(C) 2016 by the authors; licensee MDPI, Basel, Switzerland. This article is an open access article distributed under the terms and conditions of the Creative Commons Attribution (CC-BY) license (http:/ / creativecommons.org/licenses/by/4.0/). 